\title{
A self-organized metaheuristic approach towards inter-cell interference management for LTE-Advanced
}

\author{
Adnan Shahid ${ }^{1}$, Saleem Aslam', Sarmad Sohaib², Hyung Seok Kim and Kyung-Geun Lee ${ }^{\text {** }}$
}

\begin{abstract}
In order to meet the high throughput demand set by international mobile telecommunication union, carrier aggregation is exploited for expanding the bandwidth of up to $100 \mathrm{MHz}$ in Long Term Evolution-Advanced (LTE-A). For achieving the aforementioned bandwidth, a maximum of five component carriers (CCs) can be aggregated as per Release 10 LTE-A. Improper CC selection and scheduling will result in hazardous throughput, fairness, and interference problems. Therefore, a proper CC selection and scheduling algorithm is most important for the overall performance of the network. On the other hand, due to an increased information exchange, centralized network planning is not a suitable choice here. In this study, we are employing a self-organized particle swarm optimization (PSO)-based joint component carrier selection and scheduling (JCCS) algorithm for the downlink. More precisely, the concern of the proposed algorithm is the autonomous distribution of resource blocks (RBs) from the pool of CCs by the base station, with the concern of minimizing the impact of inter-cell interference. Our proposed PSO-based JCCS algorithm results in the maximization of the min-max throughput by managing the inter-cell interference in an appropriate manner. Moreover, the proposed algorithm is compared with the traditional CC selection and scheduling algorithms, i.e., random, round robin, and proportional fair. The comparison is carried out in terms of throughput and fairness, and the calculated percentage gain in the end elaborates the performance improvement by exploiting PSO-based JCCS.
\end{abstract}

Keywords: Self-organization; Particle swarm optimization; Resource blocks; Component carrier; LTE-Advanced

\section{Introduction}

Long Term Evolution-Advanced (LTE-A) is an evolving standard that will give the next generation mobile networks high data rates of $1 \mathrm{Gbps}$ for low mobility and 100 Mbps for high mobility [1]. Two international bodies, a third generation partnership project (3GPP) and an IEEE 802.16 working group, are enthusiastically working together to achieve the goals set by the international telecommunication union (ITU) in terms of an international mobile telecommunication union (IMT-A). Therefore, 3GPP started a new study item: LTE-A in March 2008 which was confirmed as an IMT-A technology in November 2010 [2]. LTE-A Release 10 is an extension of LTE Release 8/9 and provides many advantages over

\footnotetext{
* Correspondence: kglee@sejong.ac.kr

'Department of Information and Communication Engineering, Sejong University, Seoul 143-747, Republic of Korea

Full list of author information is available at the end of the article
}

LTE such as throughput, spectral efficiency, capacity, power consumption, etc. These enhancements are to be met by exploiting many exciting wireless technologies like multi-antenna, orthogonal frequency division multiplexing (OFDM) in downlink and single-carrier frequency division multiple access (SC-FDMA) in the uplink. In order to increase the bandwidth, in LTE-A, the concept of carrier aggregation (CA) is exploited for escalating the bandwidth from 20 to $100 \mathrm{MHz}$ [3].

In $\mathrm{CA}$, the bandwidth is expanded by aggregating multiple component carriers (CCs) either contiguously or non-contiguously. CA should strictly follow the $3 \mathrm{G} \mathrm{LTE}$ specifications in order to keep backward compatibility with previous releases of LTE. Figure 1 shows three different types of CA scenarios that have been proposed for LTE-A as in [4]. The three CA scenarios are intraband contiguous $\mathrm{CA}$, intra-band non-contiguous $\mathrm{CA}$, and inter-band non-contiguous $\mathrm{CA}$. The CCs belong to 


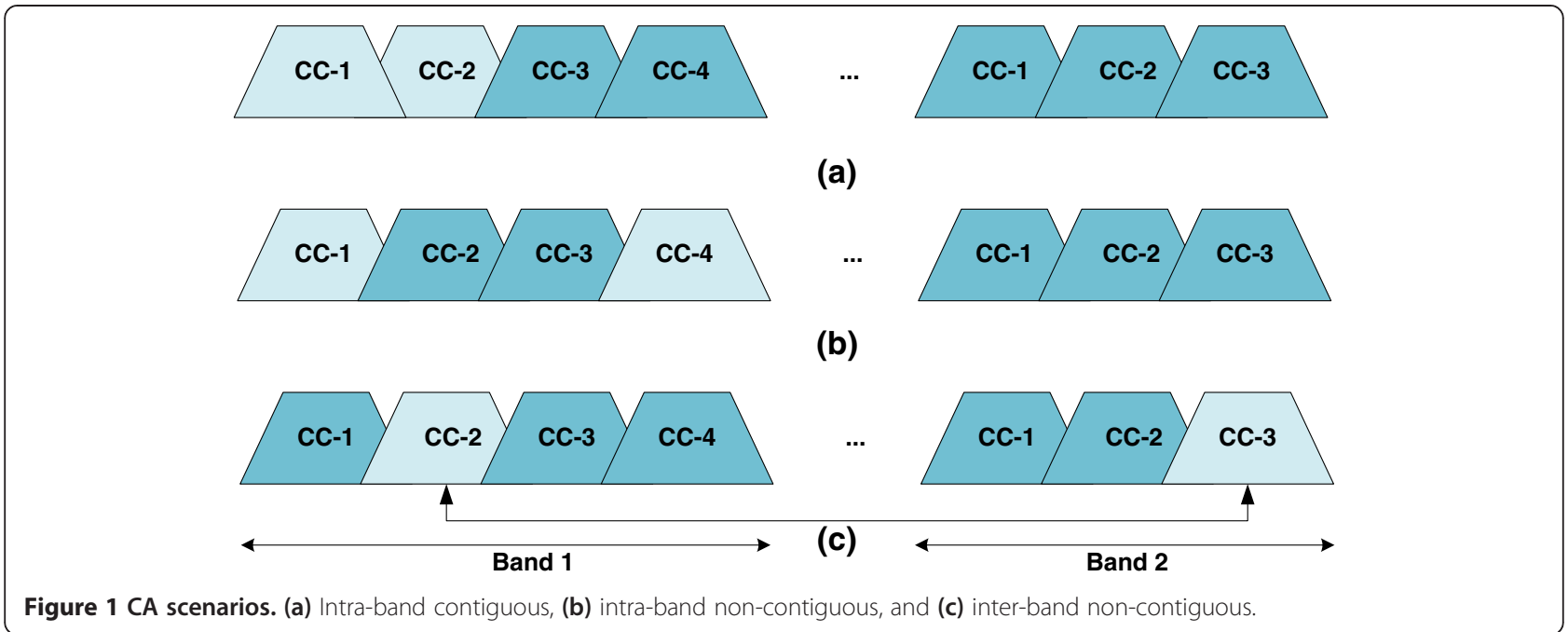

the same frequency band adjacently in the first scenario and non-adjacently in the second. In inter-band CA, the CCs belong to the different bands. In this study, we are considering all the CA scenarios and we are aiming to propose the combined optimum solution. Moreover, we are targeting CA for downlink because the concept of $\mathrm{CA}$ is more pronounced in downlink rather than uplink.

Principally, the job of the base station (eNB) is to allocate the CCs to the user equipments (UEs) based upon the instruction from the network side and also from the reporting of UEs. Various channel estimation techniques can be employed to get information about the interference and other things [5]. In order to inculcate the self-organization concept within this framework, each eNB carries out the allocation task autonomously by only having the local acquired information. This selforganization helps in allocating the resources without any involvement of the centralized entity. The aggregated carriers for CCs are of different bandwidth such as 1.4, 3, $5,10,15$, or $20 \mathrm{MHz}$, and the maximum of only five can be aggregated as per LTE-A. Hence, the maximum bandwidth in LTE-A extended up to $100 \mathrm{MHz}$. CA enables the UE to be configured on multiple CCs simultaneously, where each CC has different radio characteristics as described in the last paragraph. This will open a new research arena regarding how to distribute the CCs among UEs optimally for balancing the load across CCs and performance improvement. The multicarrier protocol structure of an LTE-A system is illustrated in Figure 2. Initially, the eNB decides which UE is to be serviced, and then layer3 CC selection allocates CCs to the UE depending upon its requirement (throughput demand, type of information, etc). After CC selection is done, packet scheduling is executed for each CC which decides the group of RBs allotted to the UE. The combination of CC selection at layer- 3 and scheduling at layer- 2 is termed as radio resource management, which is an important part of eNB in LTE-A and is a worth investigating research area that we are investigating here.

The increasing number of UEs and traffic in the future 4G cellular system leads to an unfeasible centralized network planning because of the increased overhead [6]. A self-organization-based optimization is the target for the future cellular networks that contribute to the reduction of human intervention in network planning. This is the novel concept that reduces the system capital and operational expenditure (CAPEX/OPOEX) [7]. Within the last decade, significant attention is given to the investigation of self-organization in cellular network from the academia as well as from the industry. The future cellular network is expected to provide full broadband wireless service with efficient resource utilization by employing self-organization paradigm. In this study, we are proposing a joint $\mathrm{CC}$ selection and scheduling algorithm in a self-organizing manner by exploiting a particle

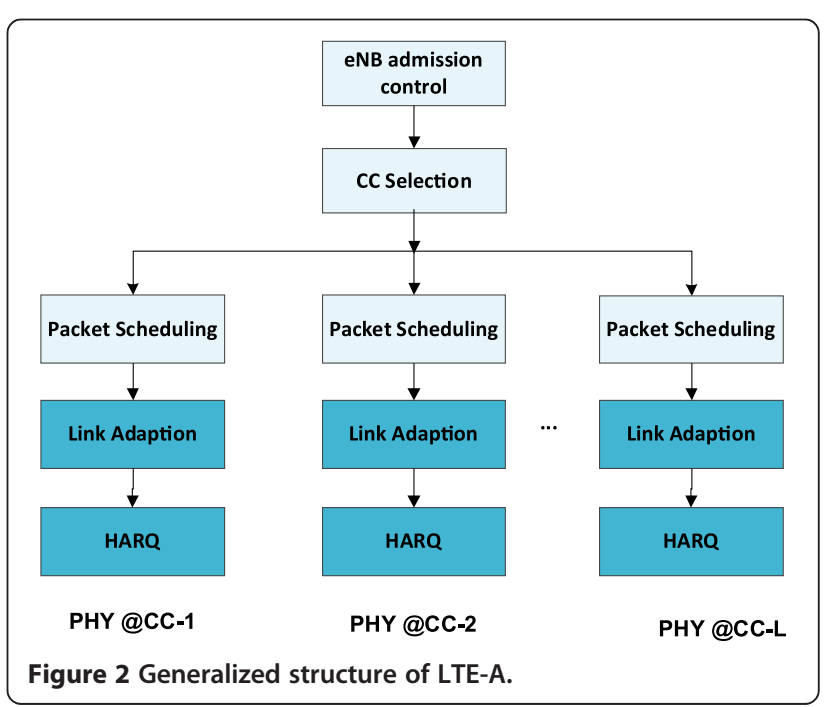


swarm optimization (PSO). The main contributions of our study are categorically described as the following:

- A self-organized algorithm is presented as the JCCS in the downlink with the concern of minimizing the impact of inter-cell interference.

- PSO, a meta-heuristic algorithm, is exploited here for distribution of RBs among the pool of CCs to UEs. The proposed algorithm is applicable for both intra-band and inter-band CA scenarios.

- A concept of CA is utilized here which corresponds to the aggregation of contiguous and non-contiguous CCs.

- The joint CC selection and scheduling (JCCS) is studied here, which has not been investigated so far.

- The analysis in terms of the maximization of min-max throughput is carried out. Additionally, the comparison is illustrated out in terms of mean throughout and fairness with other traditional methods, which justify the applicability of the proposed algorithm. Finally, the percentage gain is also evaluated.

The rest of this paper is organized as follows: Related work is outlined in Section 2. Section 3 presents the system model with the inclusion of problem formulation. The generalized PSO algorithm is presented in Section 4. Section 5 houses the algorithmic details of self-organized PSO-based JCCS. The simulation results and analysis is listed in Section 6, which contains preliminary analysis, min-max throughput, and the comparison of our proposed algorithms with the traditional ones. Finally, Section 7 concludes the article.

\section{Related work}

Recently, many researchers have indulged in investigating the issues related to designing the packet scheduler (PS) at the layer-2 as shown in Figure 2. The studies done designing PS, and carrier load balancing methods are listed in [8-13]. The assumptions made in all these papers are that the UE is assigned either just one CC [8-10] or all CCs $[11,12]$. However, in a real scenario, specifically in downlink, assigning on CC or all CCs to each UE will not meet the requirements set by the ITU. Furthermore, assigning all CCs to the UE results in severe inter-cell interference problems. In [13], the authors proposed autonomous component carrier selection algorithms, but that is relevant to the local environment. Our study is pertinent to the CC selection and scheduling of RBs to the UEs in the macro environment. To the best of our knowledge, no major work has been done in the $\mathrm{CC}$ selection domain at layer-3, specifically the combined selection and scheduling that we are going to examine in this study. Therefore, we targeted a joint approach and intend to propose an optimal solution that supersedes the other proposed ones.

The algorithms proposed for CC selection [14-16] are listed here. A G-factor based CC selection algorithm is proposed in [14]. This algorithm targeted the CA of inter-band non-contiguous CCs. It takes into account both radio channel characteristics and traffic load. In [15], the authors proposed the received signal power based CC selection algorithm. They targeted the non-continuous carrier aggregation. The traditional algorithms for CC selection and scheduling that we are going to use for comparison are random, round robin, and proportional fair. As we are proposing the joint approach, therefore, we are treating this selection and scheduling algorithms to be the same, just for comparison. In random selection/scheduling (RS), the eNB randomly allocates the resource blocks (RBs) from the pool of CCs with the goal of equalizing the UEs on each CC. The round robin (RR) concerns evenly distributing the load to all CCs with the constraint of allocating a least number of CCs to the targeted UE. Both the above listed techniques are not suited for noncontinuous CA scenarios. The authors in [16] propose an autonomous CC selection for the femtocell network. However, they employed the expected interference management rather than the acquired information that we are exploiting in this study.

Various resource allocation methods have been proposed in the literature that can mitigate inter-cell and intra-cell interference and, consequently, can improve the system performance [17-23]. The authors in [17] propose a conjunction of dual polarization and time domain resource allocation technique. However, it also includes the changing of the polarization whereas, we are targeting the resource allocation that can mitigate inter-cell in a selforganizing manner and improves system performance. The authors in [18] propose an adaptive resource allocation scheme for minimizing the transmit power. However, only considering the power minimization leads to violation of quality of service (QoS) requirement in certain cases. There has been some work in the self-organization for the wireless systems. The authors in [19] propose a distributed self-organization by exploiting Gibb's sampler concept. However, they considered potential delay for throughput optimization. The authors in [20] present a heuristic approach for multiuser scheduling. The results illustrate that the implication of heuristic approaches gives near optimal results at the cost of bearable complexity. The authors in [21] propose a heuristic approach for resource management in femtocell networks. A docitive learning mechanism for resource allocation and power control is presented in [22], where the femtocell environment is considered. However, the concept of CA is not taken into account which is the state of the art technology for escalating the system performance. The authors in [23] 
propose a downlink resource management by combining multiuser MIMO and CA. However, we are considering the joint optimization in terms of RB and CC selection.

According to the best of authors' knowledge, a selforganization scheme for PSO-based JCCS has not been investigated so far. The proposed algorithm mitigates intercell interference and provides a near optimal solution at cost of bearable computation complexity. An important point of the proposed algorithm is that the selforganization concept has been exploited in which the eNB carries out the scheduling task in an autonomous manner.

\section{System model}

\subsection{Proposed framework}

The proposed framework of self-organized PSO-based JCCS within LTE-A is illustrated in Figure 3. The information about the RBs is acquired by each eNB from their connected UEs based upon the direction of eNB. Moreover, this information includes the received signalto-interference-plus-noise ratio (SINR) on different channels and is conveyed via channel quality indicator (CQI) on S1 interface, where S1 is the interface between the UEs and network components in the context of LTE standard. These measurements quantitatively differentiate the RBs from the pool of CCs in terms of interference in different geographical regions of the cell. The geographical information of the UEs is acquired by the network entities mobility management utility (MME) and evolved serving location center (E-SMLC). This gathered information is exploited in our proposed algorithm for carrying out the scheduling task in a self-organizing manner. The proposed PSO-based JCCS algorithm is represented by the block in Figure 3. Specifically, we are taking into consideration the inter-cell interference with the assumption that intra-cell interference is not there. This assumption is based upon the fact that one RB can only be utilized by a single UE within a cell, and also a strict synchronization in the OFDMA-based subcarriers is assumed here. The concern of this tight synchronization assumption means that interference is only inculcated if there is a transmission on same RBs. The general theme of the proposed algorithm is to iteratively distribute the RBs in a self-organizing manner with the concern of minimizing the interference or maximizing the system performance. In order to reduce the information exchange for the reporting of the RBs, single CQI is utilized for a group of RBs. In addition, on each $\mathrm{eNB}$, the average of CQI values is computed for the geographically close UEs and this is done for having the most reliable information. The two benefits of exploiting this self-organized joint approach are: firstly, each eNB carries out the scheduling task based upon the local information received from the directed UEs and secondly, by employing the joint approach efficient spectrum, utilization is achieved based upon reducing the inter-cell interference.

\subsection{Problem formulation}

We consider a downlink CA in LTE-A with a frequency reuse-1, where there are $N$ UEs in different cells. Moreover, there are $M$ RBs within $L$ CCs. Each UE can be operated on multiple CCs simultaneously. However, the number of CCs that the UE has to connect should be as small as possible considering the power saving and signal processing complexities. According to the 3GPP LTE-Advanced specifications, the maximum number of five CCs can be allocated to UE. This corresponds to the fact that the maximum bandwidth of the UE cannot be

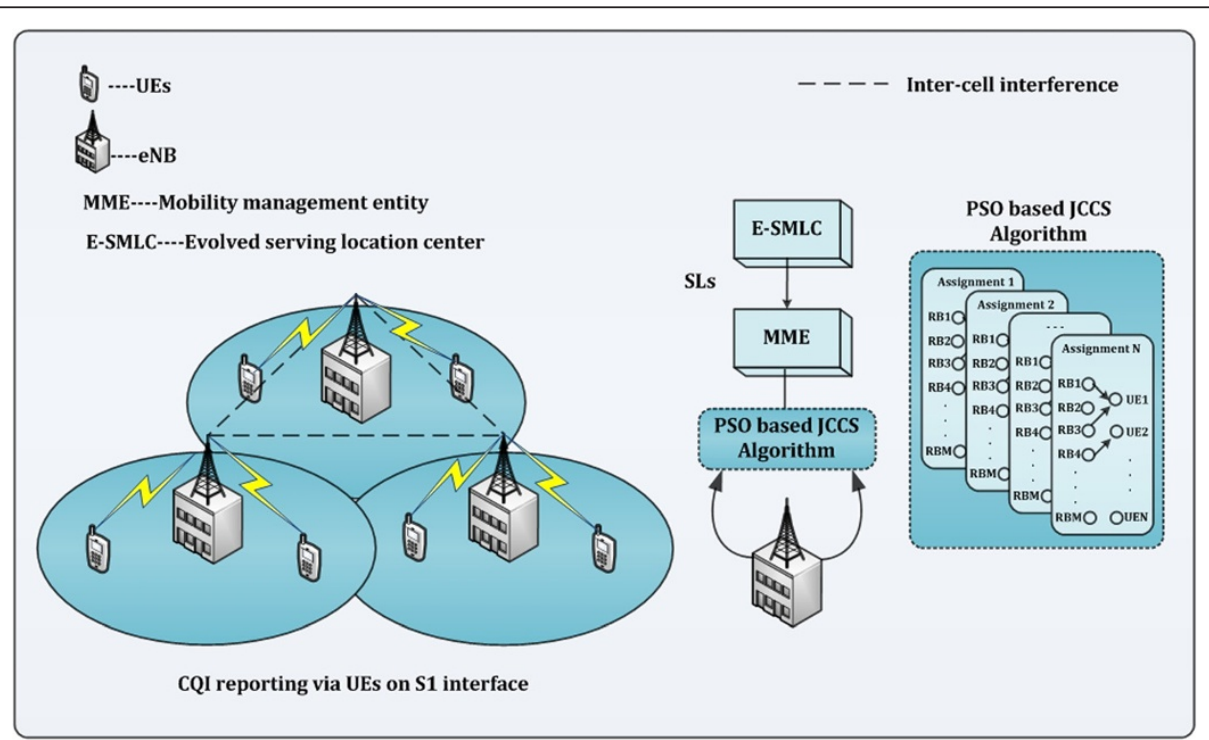

Figure 3 Proposed framework. 
exceeded beyond $100 \mathrm{MHz}$. Particularly, in this study, we are considering that the number of CCs allocated should be in accordance to the 3GPP LTE-Advanced specifications. Each CC is divided into many RBs, and these are grouped into 12 adjacent subcarriers with an inter-subcarrier spacing of $15 \mathrm{kHz}$ as shown in Figure 4. Each RB comprises of six or seven OFDM symbols and spans a length of $0.5 \mathrm{~ms}[24,25]$. In this study, we are taking into account frequency division duplex (FDD) downlink transmission and resource scheduling. Within LTE-Advanced downlink, a UE can be allotted a number of RBs; however, for a single cell, a RB cannot be assigned to more than one $\mathrm{UE}$ as in (5). Additionally, strict synchronization is assumed here which means that interference is only induced when there is a transmission of two network entities on same RB in parallel. Thereby, the only interference component that exists here is the intercell which has to be managed.

The proposed algorithm is applicable for both intra-band and inter-band $\mathrm{CA}$ scenarios. Concerning the case of $\mathrm{CA}$ in the downlink, consider a set of $L \mathrm{CCs}\left\{\mathrm{CC}_{1}, \mathrm{CC}_{2}, \mathrm{CC}_{3}, \ldots\right.$, $\left.\mathrm{CC}_{L}\right\}$ with the further variable division of RBs within each CC. This variable distribution is represented by $\mathrm{M}_{l}$, which corresponds to the number of RBs in $l$ th CC. Each CC is assumed to be of different bandwidth for better analyzing the real situation, and the goal is to distribute among UEs based upon the variable throughput demand. Moreover, it is also assumed that our system consists of $N$ UEs such as $\left\{\mathrm{UE}_{1}, \mathrm{UE}_{2}, \mathrm{UE}_{3}, \ldots, \mathrm{UE}_{N}\right\}$. Also, the $K$ base stations are represented by a set $\left\{\mathrm{eNB}_{1}, \mathrm{eNB}_{2}, \mathrm{eNB}_{3}, \ldots, \mathrm{eNB}_{K}\right\}$. In this study, we are considering the frequency reuse- 1 system. However, the results can be extended to any system.

The transmission power of $k$ th eNB on $m$ th RB from $l$ th $\mathrm{CC}$ is represented as $P_{m l}^{k}$. In addition, we are assuming that if the eNB is not transmitting on the RB, then $P_{m l}^{k}=0$. As far as the power budget is concerned, we are assuming that fixed power budget is available such that $\sum_{l} P_{m l}^{k} \leq W$. In other words, this can be considered as the power constraint that each eNB has. Here we assumed that the power is uniformly distributed among the allotted pool of RBs. The path loss and shadowing effect are included in the considered channel models. Let $H_{m l}^{n k}$ be the channel gain between $k$ th $\mathrm{eNB}$ and $n$th UE on the $m$ th RB of $l$ th CC. The received power on the $n$th UE from the $k$ th eNB is $H_{m l}^{n k} l_{m l}^{k}$ and on the other UEs this is the interference components. Based upon the above mentioned concerns, the SINR of the $n$th $\mathrm{UE}$ on $m$ th $\mathrm{RB}$ from $l$ th $\mathrm{CC}$ is given by

$$
\operatorname{SINR}_{m l}^{n}=\frac{H_{m l}^{n k} P_{m l}^{k}}{\sigma_{m l}^{n}+\sum_{q \neq k}^{K}\left(\sum_{l=1}^{L}\left(\sum_{m=1}^{M_{l}} x_{m l}^{n} H_{m l}^{n q} P_{m l}^{q}\right)\right)}
$$

where $\sigma_{m l}^{n}$ is the noise that is experienced by the $n$th UE on the $m$ th RB of $l$ th CC, $x_{m l}^{n}$ is the decision variable that corresponds to the assignment of $m$ th RB from $l$ th $\mathrm{CC}$ to $n$th UE and is defined in Equations (9) and (11). The achieved throughput by $n$th UE on the allocated RBs is given by

$$
\gamma^{n}=\sum_{l=1}^{L}\left(\sum_{m=1}^{M_{l}} x_{m l}^{n} B \log _{2}\left(1+\operatorname{SINR}_{m l}^{n}\right)\right)
$$

The number of RBs to be allotted to the $n$th is based upon the demand $D_{n}$ in terms of number of RBs. In order to minimize the inter-cell interference and to achieve the increase throughput performance, the problem function can be formulated as:

$$
\text { Maximize: } \sum_{n=1}^{N} \gamma^{n}
$$

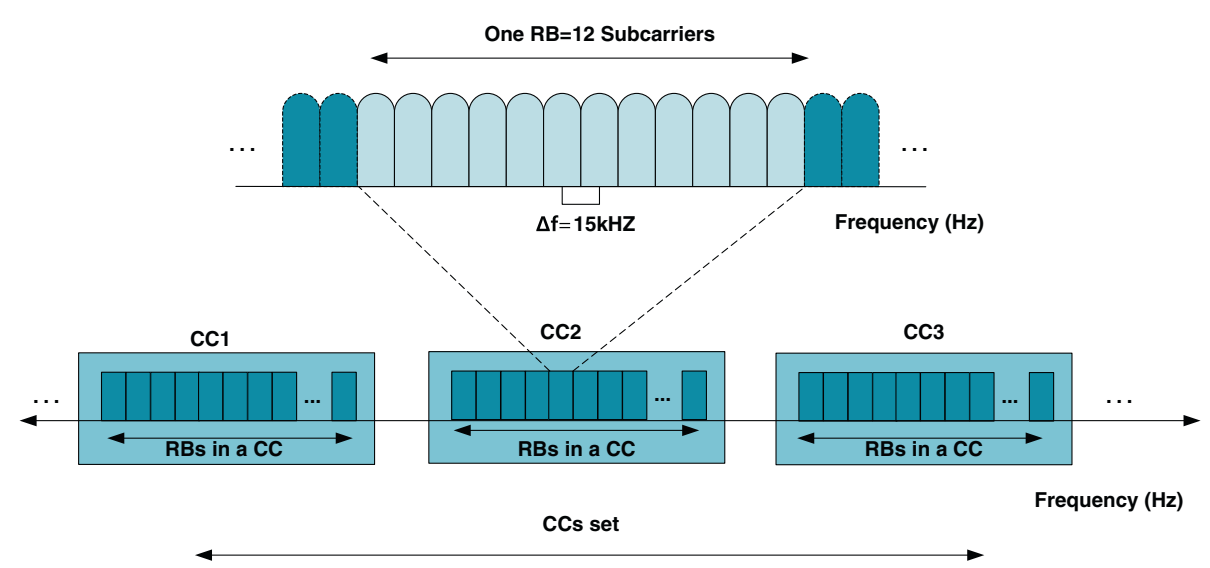

Figure $4 \mathrm{RBs}$ and $\mathrm{CCs}$. 


$$
\begin{aligned}
& \text { Subject to: } \sum_{l=1}^{L} \sum_{m=1}^{M_{l}} x_{m l}^{n}=D_{n}, \forall n=\{1,2,3, \ldots, N\} \\
& x_{m l}^{n 1} \neq x_{m l}^{n 2}, \forall n 1 \neq n 2, k \\
& \gamma^{n} \geq \Gamma_{0}, n=\{1,2,3, \ldots, N\} \\
& \sum_{l=1}^{L} \sum_{m=1}^{M_{l}} P_{m l}^{k} \leq W, \forall k=\{1,2,3, \ldots, K\} \\
& P_{m l}^{k} \geq 0, k=\{1,2,3, \ldots, K\}, m=\left\{1,2,3, \ldots, M_{l}\right\} \\
& \quad l=\{1,2,3, \ldots, L\} \\
& \quad l=\{1,2,3, \ldots, L\} \\
& x_{m l}^{n}=\{0,1\}, n=\{1,2,3, \ldots, N\}, m=\left\{1,2,3, \ldots, M_{l}\right\} \\
& \sum_{l=1}^{L} \frac{x_{m l}^{n}}{M_{l}} \leq 5, \forall n=\{1,2,3, \ldots, N\}, m=\left\{1,2,3, \ldots, M_{l}\right\}
\end{aligned}
$$

The formulation comprises of important constraint that the UE be allotted a number of RBs from its acquired demand as in (4). Additionally, a minimum throughput criterion is also included as in (6) for limiting the effect of greedy behavior of other eNBs UEs. By maximizing the throughput that is achieved (3), inter-cell interference can be significantly reduced, under the assumption that intra-cell interference is not present. This is due to the fact that two different UEs in a same cell cannot be allotted a same RB as in (5). In addition, the number of CCs allocated should be in accordance to 3GPP LTEAdvanced specifications as in (10). Hence, the only interference that exists in our case is the inter-cell. Equation 9 is the decision variable and is represented as:

$x_{m l}^{n}=\left\{\begin{array}{l}1 ; \text { if the } m \text { th RB of } l \text { th } C C \text { is assigned to } n \text {th UE } \\ 0 ; \text { if the } m \text { th RB of } l \text { th } C C \text { is not assigned } t o n \text {th UE }\end{array}\right\}$

\subsection{Notations and assumptions}

The notations presented in Table 1 are used in the rest of the paper.

\section{Particle swarm optimization}

PSO is a population-based bio-inspired algorithm inspired by the bird flocking and fish schooling mechanism. These types of algorithms are specifically useful where the sample space is very large, the parameters of interest are dynamic, and there is not too much information exchanged between the users (particles) [26].
Table 1 Notations and assumptions

\begin{tabular}{ll}
\hline Parameters & Meaning \\
\hline$K$ & Total number of eNBs \\
$L$ & Number of UEs in the system; \\
$M_{j}$ & Total number of CCs; \\
$P_{m l}^{k}$ & Total number of RBs in /th CC; \\
$W$ & Transmission power on $k$ th eNB on $m$ th RB of Ith CC; \\
$H_{m l}^{n k}$ & Total power budget available on $k$ th eNB; \\
$S I N R_{m l}^{n}$ & Channel gain between $k$ th eNB and $n$th UE operating \\
$x_{m l}^{n}$ & On mth RB of lth CC; \\
$\gamma^{n}$ & SINR value at the $n$th UE on $m$ th RB of lth CC; \\
$D_{n}$ & Decision variable as described in (9); \\
$\Gamma_{0}$ & The achieved capacity by the $n$th UE; \\
$P$ & The demand in terms of RBs of the $n$th UE; \\
$X_{j}$ & The minimum capacity throughput limit in the system; \\
$V_{j}$ & Particle size in the proposed algorithm; \\
\hline
\end{tabular}

The PSO algorithm starts with populating the swarm of particles, where each particle represents a potential solution. The swarm is similar to the population while a particle is equivalent to the individual. Each particle is associated with a position and velocity in search space. In each iteration of the algorithm, the specified fitness is computed and both the velocity and position of each particle are updated according to (11) and (12), respectively. The velocity of each particle is updated according to the finest two known positions, personal best position (pbest) and the neighborhood best position (nbest), where pbest is the best position the particle has visited and nbest is the finest position corresponding to the particle, and its neighbor-hood have visited since for the first time step. When the whole swarm is considered as the neighbor, then the nbest is termed as the global best (gbest) and for a small neighborhood, nbest is equivalent to the local best (lbest). The major difference between the two positions is the convergence, i.e., due to large particle size: gbest PSO converges faster than the lbest. Furthermore, lbest has the chance of being trapped because of a small sample space. Therefore, in our case, we are using gbest due to its yielding faster convergence.

$$
\begin{aligned}
V_{j}^{\text {new }}= & V_{j}+c_{1} r_{1}\left(\text { pbest }_{j}-X_{j}\right) \\
& +c_{2} r_{2}\left(\text { nbest }_{j}-X_{j}\right) \ldots j=1,2, \ldots, P \\
X_{j}^{\text {new }}= & X_{j}+V_{j}^{\text {new }}
\end{aligned}
$$

where $c_{1}$ and $c_{2}$ are termed as acceleration coefficients whose job is to control the influence in the search 
process; $P$ is the total number of particles in a swarm; $r_{1}$ and $r_{2}$ are two random numbers and are uniformly distributed in the interval from 0 to 1 ; and $X j, X_{j}^{n e w}, V j$, and $V_{j}^{\text {new }}$ represent the current and updated position and velocity of the $j$ th particle.

Figure 5 shows the generalized work of PSO in a flow chart format [27].

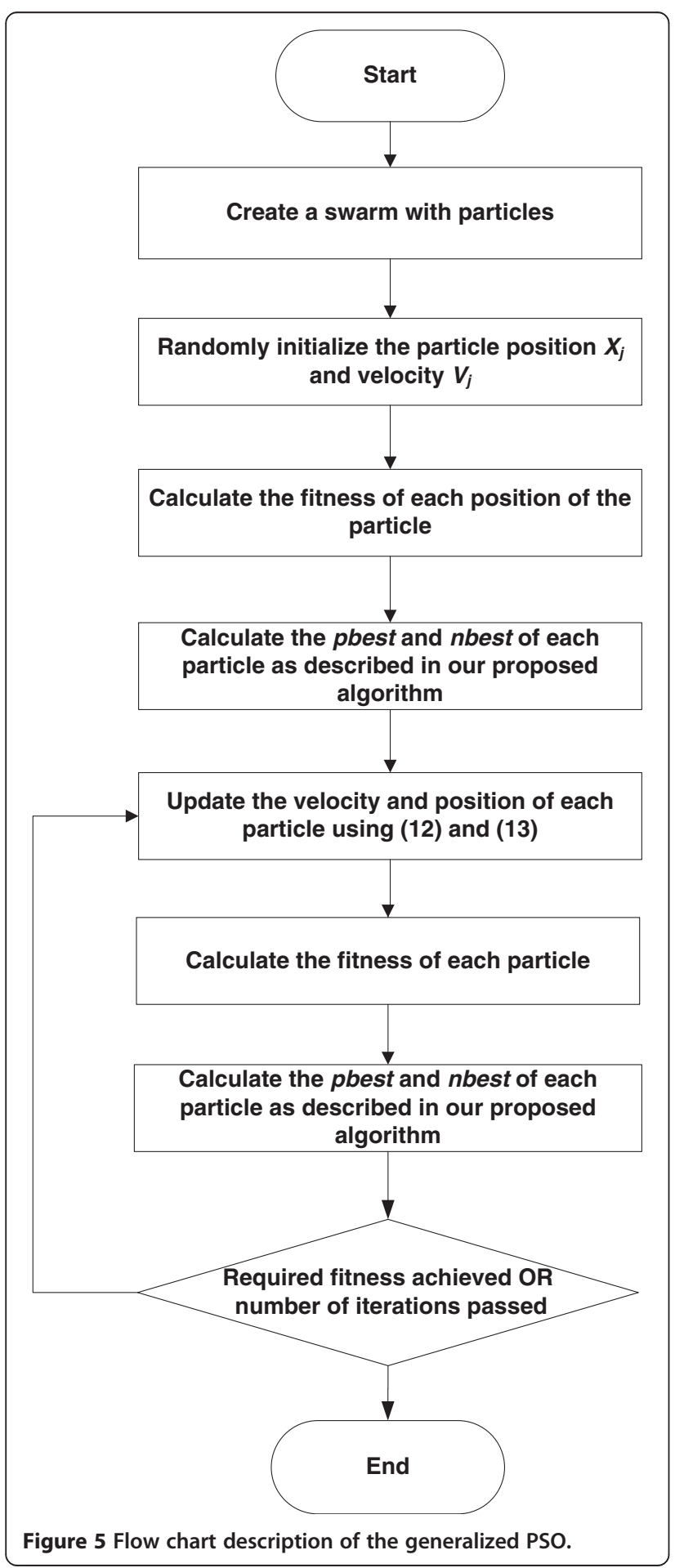

\subsection{Convergence analysis}

The investigation of convergence characteristic of any optimization problem is of paramount importance. Thereby, it is necessary to analyze the convergence characteristic of PSO before giving a practical solution. The analysis of the convergence characteristics of the PSO is a tiring task because of the stochastic nature of PSO. Two conditions, $H 1$ and $H 2$ given in [28], help in proving the convergence of the optimization algorithm.

Given the optimization algorithm $A$, the optimization problem $(B, f), X_{i}$ is the result of $i$ th iteration, and the next iteration $X_{i+1}\left(X_{i+1}=\mathrm{A}\left(X_{i}, \Omega\right)\right)$, where $\Omega$ is the sorted out solution by the algorithm $A$. The condition $H 1$ can be expressed as:

Condition H1: $f(A(X, \Omega)) \leq f(X)$, if $\Omega \in B$, then we have $f\left(A\left(X_{1}, \Omega\right)\right) \leq f(\Omega)$ where $B$ is the constraint space of the problem under consideration.

The concern of the aforementioned condition stipulates that the new solution given by $A$ is no worse than the current solution. The global convergence is defined as the sequence $\left\{f\left(X_{i}\right)\right\}_{i=0}^{\infty}$ can reach to the infimum, where inf $(f(X \in B))$ is the viable solution in $B$. On the other hand, it is possible that the viable solution $B$ comprises of isolated spots, infimum, and discontinuous spaces. Thereby, considering this potential problem, the search infimum is defined by Lebesgue measure space.

$$
\Phi=\inf (s: q[X \in B \mid f(X)<s]>0)
$$

Where $q[X]$ denotes the Lebesgue measure in set $X$. The definition of $q[X]$ and $B$ guarantees that the nonempty point does not contain in set $B$. In other words, the optimization algorithm can reach or close to the infimum instead of searching all points. The algorithm can be considered to find a solution (global optimum or near global optimum) if it can generate the point in the optimality region $R_{0}$ defined as:

$$
R_{0}=\{X \in B \mid f(X)<\Phi+\varepsilon\}
$$

where $\varepsilon>0$.

Condition H2: A sufficient condition for the global optimum can be written as:

$$
\prod_{i=0}^{\infty}\left(1-q_{\mathrm{i}}[E]\right)=0, \forall E \subseteq B, q[E]>0
$$

where $q_{i}[E]$ is the probability measure generated by $q_{i}$.

Theorem 1: Assuming that $f$ is a measurable and viable solution, the algorithm $A$ satisfies the aforementioned conditions $H 1$ and $H 2$. If the sequence generated by the algorithm $A$ is $\left\{f\left(X_{i}\right)\right\}_{i=0}^{\infty}$, then

$$
\lim _{i \rightarrow+\infty} U\left[X_{i} \in R_{0}\right]=1
$$

The proof of the theorem is given in [28]. In order to achieve the global convergence, the stochastic optimization 
algorithm must satisfy conditions $H 1$ and $H 2$. The considered PSO satisfy both the conditions and can achieve the global convergence.

\section{Self-organized PSO-based JCCS algorithm}

The proposed self-organized algorithm is described in three different steps. First, each eNB directs the UEs on the control to send the CQI for the group of RBs. Second, UE sends the corresponding CQI values as directed by the eNB. Third, based on the received information, the self-organized PSO-based JCCS algorithm is carried out, which iteratively select the best solution information within the constraint described in Equations (4) to (9). An important aspect of the proposed algorithm is that it only relies on the local received information with any central entity for carrying out the scheduling task. The objective of the optimization is to maximize the capacity based upon the demands of the UE. The detail of proposed algorithm is presented hereafter.

\subsection{Algorithm}

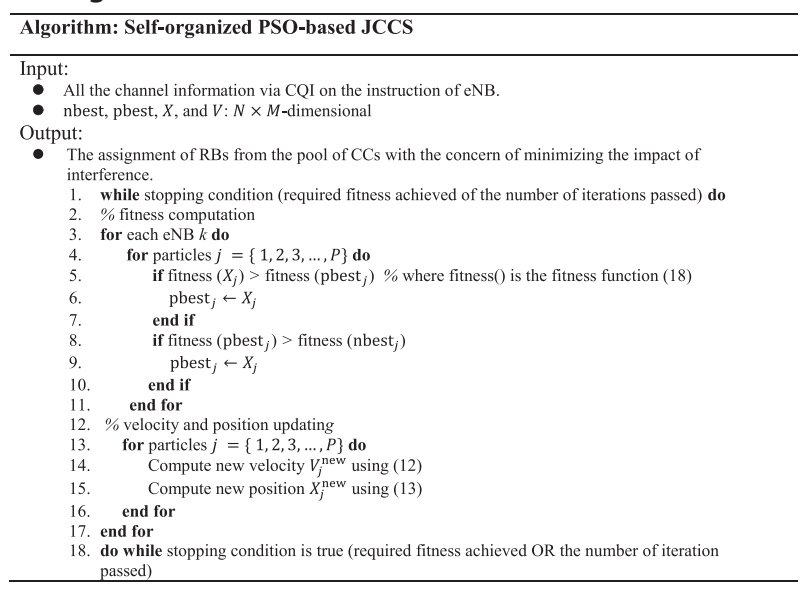

\subsection{Particle encoding}

A major concern in PSO being workable for the CC selection and RBs assignment is to map a problem solution and a particle. In this paper, every particle position is represented in a $M$-dimensional space, where $M$ is the total number of RBs in $L$ CCs. The particle's position in $M$-dimensional space is filled with a positive integral number in the range from 1 to $N$. The $X_{j}=\left\{X_{j, m l}, \ldots, X_{j, m l}\right\}$ represents the position of $j$ th particle, and $X_{j, m l}$ is the assignment of $m$ th RB of $z$ th CC to the UE which is being scheduled by the proposed algorithm.

\subsubsection{Fitness computation}

Fitness value depicts the excellence of the particle's solution in PSO. In this study, we are using the fitness as maximization of the throughput under the networks constraints (4) to (10). The fitness function aims at maximizing the throughput in a self-organizing manner which indirectly minimizes the inter-cell interference. Furthermore, in order to avoid the performance deterioration of neighbor cells, a minimum capacity check (6) is inculcated in the problem formulation. In the proposed PSO-based JCCS, in each iteration of the PSO, new position and velocities are updated which results in achieving better solutions in terms of good fitness values. The fitness of the jth particle is represented as:

$$
\text { fitness }[j]=\max \left(\frac{1}{N} \sum_{n=1}^{N} \gamma^{n}\right)
$$

\subsubsection{Velocity and position changing}

The velocity and position of each particle $j=\{1,2,3, \ldots, P\}$ is updated as described in algorithm in Section 5.1. Each new particle's position results in better solution in terms of high capacity value. Therefore, the general theme of this iterative algorithm is to minimize the inter-cell interference and to give optimum assignment of the RBs from the pool of CCs. The pbest, gbest, and fitness calculation in each iteration results in changing the particle velocity appropriately and the same with the position. The pbest and gbest are being updated in a similar fashion as well as particle initialization and is elaborated in the algorithm- 1 . The velocity matrix is of dimension $N \times M$, in which all the elements are real numbers in the interval $[-N, N]$. The bound of velocity matrix of $j$ th particle is represented by

$$
V_{j} \in\left[-V_{\max }, V_{\max }\right] \quad \forall\{j=1,2,3, \ldots, P\}
$$

\subsection{Counter example}

For further elaboration, let us consider the scenario in which we have three CCs, each having a different bandwidth. The number of RBs per CCs are $C_{1}=\left\{\mathrm{RB}_{1,1}\right.$, $\left.\mathrm{RB}_{1,2}, \quad \mathrm{RB}_{1,3}\right\}, \quad \mathrm{CC}_{2}=\left\{\mathrm{RB}_{2,1}, \mathrm{RB}_{2,2}\right\}$, and $\mathrm{CC}_{3}=\left\{\mathrm{RB}_{3,1}\right.$, $\left.\mathrm{RB}_{3,2}, \mathrm{RB}_{3,3}, \mathrm{RB}_{3,4}\right\}$ and are to be distributed among two UEs, depending upon their throughput demand. Each particle is represented by nine elements $X_{j}=\left\{X_{j 1,1}, X_{j 1,2}\right.$, $\left.X_{j 1,3}, X_{j 2,1}, X_{j 2,2}, X_{j 3,1}, X_{j 3,2}, X_{j 3,3}, X_{j 3,4}\right\}$, where $X_{j l, m}$ is the allocation of $m$ th RB of $l$ th CC to UE. Figure 6a shows the position assignment of one single particle. For example, $\mathrm{UE}_{1}$ allocated six $\mathrm{RBs}$ from three $\mathrm{CC}$ s, i.e., $\mathrm{RB}_{1,1}$, $R B_{1,3}, R B_{2,1}, R B_{2,2}, R B_{3,2}$ and $R B_{3,3}$. The SINR values in $\mathrm{dBs}$ those results while allocation of $m$ th $\mathrm{RB}$ from $l$ th $\mathrm{CC}$ is also elaborated. This single shot information is just presented to have an intuitive information of the proposed PSO-based JCCS algorithm. However, in real sense, there are many RBs available and also many UEs with variable demand. Hence, different assignment results in different overall performance of the algorithm. The goal of the proposed iterative algorithm is to allocate the RBs to the UEs in a manner to increase the throughput by minimizing the impact of inter-cell interference. On the 


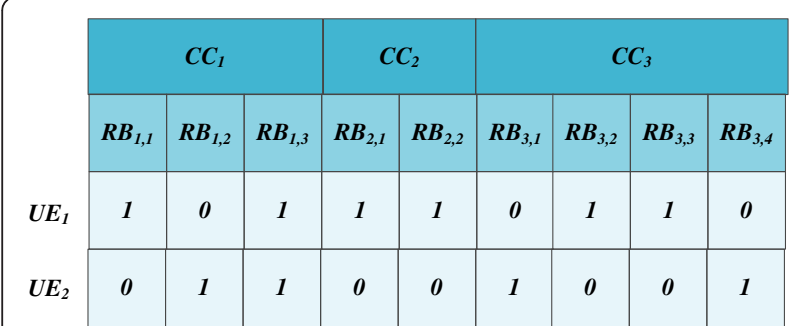

(a)

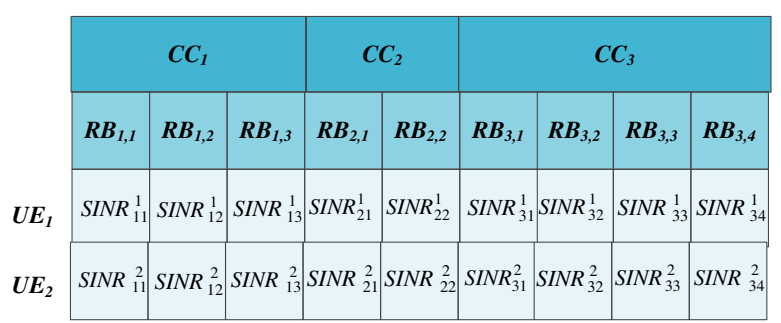

(b)

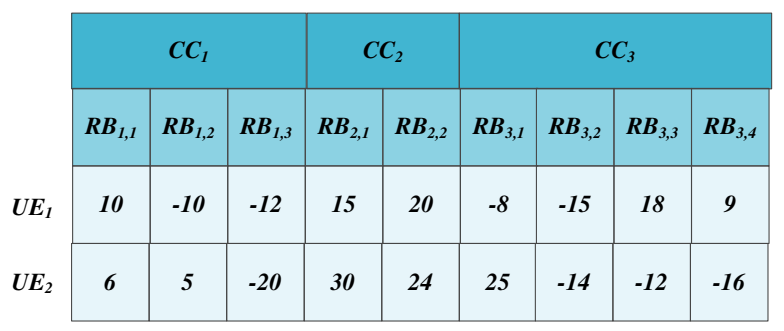

(c)

Figure 6 PSO-based JCCS algorithm. (a) Particle assignment, (b) SINR assignment generalized, and (c) SINR values in dBs.

other hand, the proposed algorithm purely relies on the local received information. In order to mitigate the greedy behavior, a minimum throughput is also included which jeopardize the violation of the performance.

As far as the complexity of the proposed algorithm is concerned, the proposed algorithm relies on the SINR values acquired from the UEs on the direction of the eNB. Once they are received, the PSO algorithm gives near optimal solution with bearable complexity under the network constraints (4) to (10). The plus point of the proposed algorithm is that it totally relies on the local received information without any involvement of any centralized entity, which significantly reduces the information among various cells and also to the network. However, the communication overhead that exists within a cell for the transfer of SINR information is minimized by using a single CQI value for a group of RBs. Additionally, not all the UEs within a cell transfer that information; it is to be decided by the concerned eNB based upon the geographical region that when the information needs to be updated. By incorporating this thing, significant information exchange overhead can be reduced. On the other hand, the proposed algorithm is also scalable in the sense that any new user can be a part of the network and is serviced by the eNB in a self-organizing manner. However, the algorithm may take time to converge as it is heuristic algorithm. But once the convergence is achieved, the results are far more superior as compared to the traditional methods as in Section 6.2. The proposed PSO-based JCCS algorithm is more viable to a scenario in which the channel effects are not changed that much. The reason is that in harsh channel environment, the RB allocation takes place repeatedly, i.e., around every half frame $(5 \mathrm{~ms})$. Therefore, for the large varying environment, the proposed PSObased JCCS will not get the near optimal results because of repeated RB allocations.

\section{Simulation results and analysis}

The analysis that we carried out is divided into three subsections: preliminary analysis, min-max throughput, and comparison. In the preliminary analysis section, the parameter selection of the proposed PSO-based JCCS algorithm is carried out. Additionally, the simulation setup regarding the WINNER II channel model is also included in this section. Different parameter values result in varying fitness values, which will have a significant impact on system performance. Therefore, it is very important to choose the appropriate PSO parameters. The first and the foremost parameter is the particle size, i.e., the initial population count. Therefore, an exhaustive search is carried out in selecting parameters like particle size, acceleration coefficients, and inertia, as they all have a significant impact on the algorithm performance. Moreover, different particle sizes have varying performances in terms of fitness values, which is also illustrated in this section. In the second section, the maximization of the min-max throughput is elaborated. Finally in the third section, we compared our proposed algorithm with traditional algorithms in terms of throughput and fairness. 


\subsection{Simulation setup}

We consider a reuse-1 FDD system with eight eNBs that share the same bandwidth of $4 \times 10 \mathrm{MHz}$. The distance between the eNBs is considered to be $500 \mathrm{~m}$. Additionally, the numbers of UEs $N$ are taken to be in the range from 40 to 80 . The convergence analysis and min-max throughput in Figures 7 and 8 are computed for $N=50$ UEs, while the comparison in Figures 9, 10 and 11 is carried out for varying UEs such that $N=40$ to 80 . As far as the number of RBs in CC set is concerned, we take $L=4 \mathrm{CCs}$ and a variable division of RBs. The total number of RBs in CCs set is considered to be $M=200$. We are employing WINNER II channel model for the system level simulations. Various propagation scenarios exist with WINNER II channel model which include indoor office, indoor-to-outdoor, stationary, rural macrocell, urban macrocell, etc. For the investigation of our algorithm, we have utilized urban macrocell model denoted as $\mathrm{C} 2$. The path loss models for the urban macrocell environment are given in Table 2 [29].

In path loss models, $d$ is the distance between the UE and the eNB, $h_{\mathrm{eNB}}$ and $h_{\mathrm{UE}}$ are the antenna height of $\mathrm{eNB}$ and $\mathrm{UE}$, respectively, $f_{c}$ is the operating frequency in $\mathrm{GHz}$, and PL is the channel gain in dBs. The power budget $W$ of each eNB is assumed to be $1 \mathrm{~W}$. The thermal noise is randomly generated in $[3.5-4.5] \times 10^{-15}$. The shadowing effect is incorporated with a mean of 0 and standard deviation of $8 \mathrm{~dB}$.

The simulation is carried out in MATLAB based upon the aforementioned simulation setup. The proposed
PSO-based JCCS is being implemented here in a selforganizing manner on each eNB. For getting the optimum solution in terms of best fitness, four particles are considered, i.e., 4, 8, 12, and 20. Moreover, the other parameters like acceleration coefficients $\left(c_{1}\right.$ and $\left.c_{2}\right)$ and inertia weight are also fine-tuned to achieve the best fitness. On the other hand, the investigation had been done in [30] that it is a good choice to set $V_{\max }=X_{\max }=N$, whenever there is lack of information regarding the $V_{\max }$ which is also true in our case. Along with the selection of the value for $V_{\max }$, an inertia weight value $w=0.6$ is also a good starting point. The selected parameters for our proposed algorithm are listed in tabular form in Table 3.

As already discussed, different particle sizes have varying effect on the fitness values. The result of employing four different particles such as 4, 8, 12, and 20 in our resource optimization problem is shown in Figure 7 . The average objective function, which is the actually the fitness in our study, is plotted with respect to number of iterations for the different particles sizes. All the four fitness curves are achieved with the same parameters such as $N=50, L=10$, and $M=250$. It can be depicted that a particle size of 12 outperforms the other particles. We have selected the particle size 12 for further analysis because of its being the best objective function value. Furthermore, on average, the selected particle size yields $20 \%$ better performance than particle sizes 4 and 20, and $4 \%$ better than particle size 8 . The rest of the analysis is based on the selected configuration.

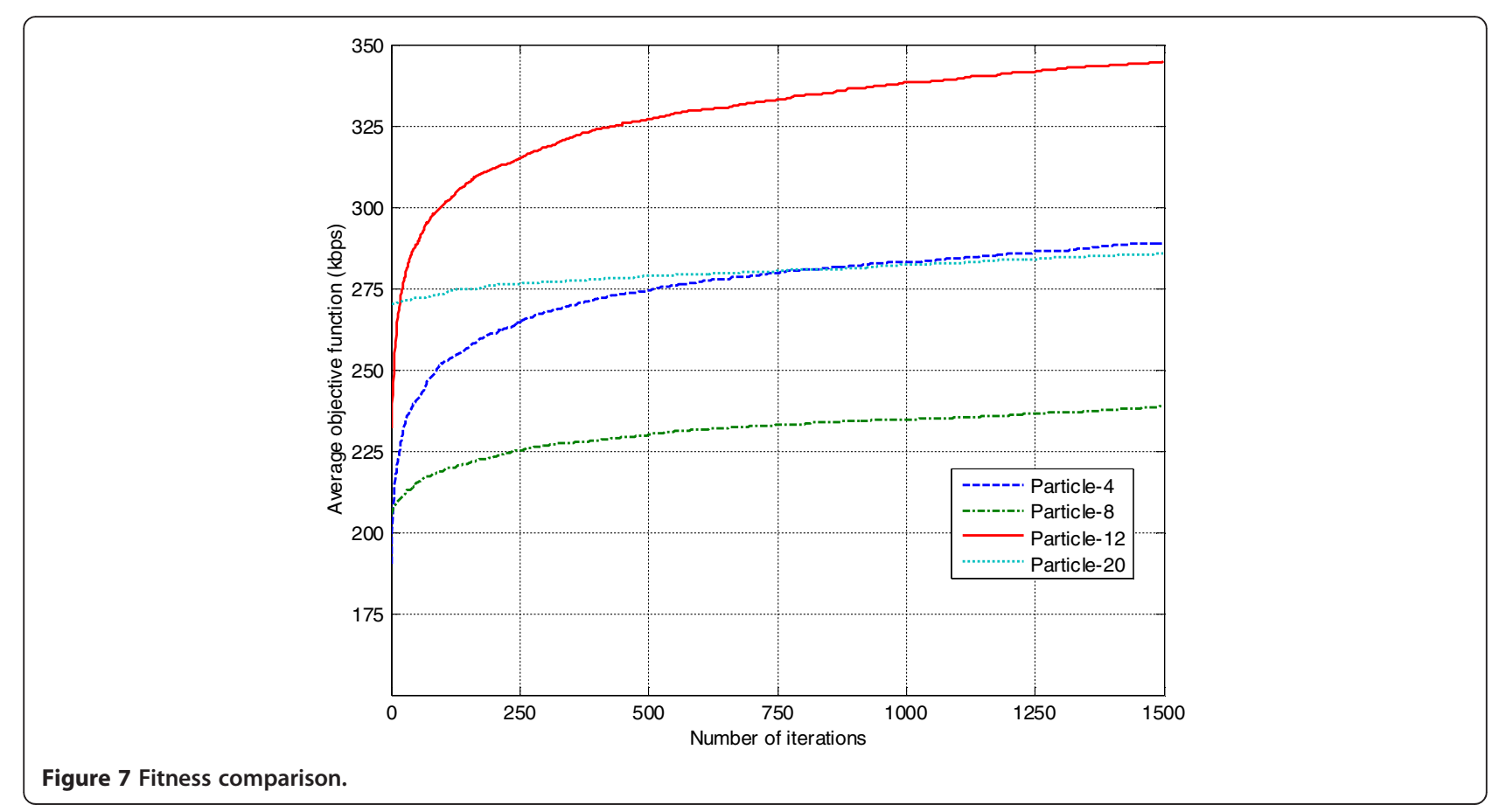




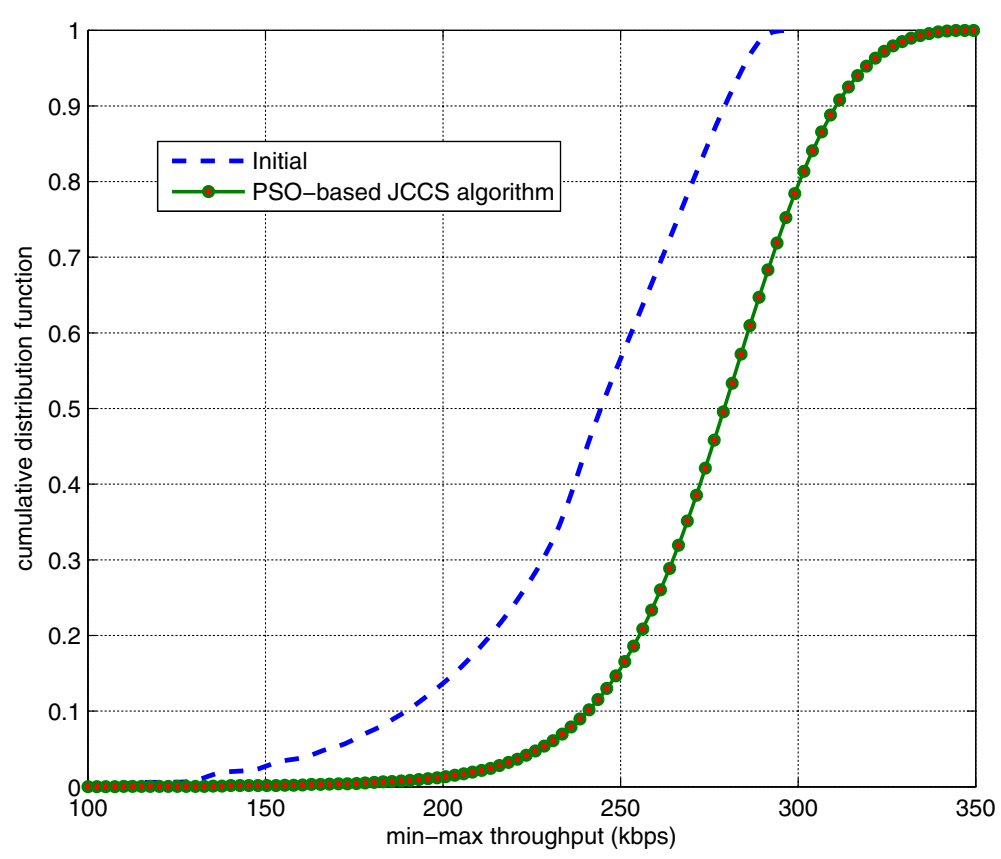

Figure 8 The cdf of min-max throughput.

\subsection{Min-max throughput}

The main theme of our proposed algorithm is the minimization of the inter-cell interference. However, the important performance edge that is gained from this optimization is the maximization of the min-max throughput. We will illustrate here how our proposed algorithm results in maximizing of the min-max throughput.

The cdf of the min-max throughput of the UEs is plotted in terms of initial population and after optimization in Figure 8. It can be seen that because of exploiting the maximization of the throughput in our proposed PSObased JCCS, the average min-max throughput of the network is enhanced. The initial population that is generated by randomly assigning the RBs, without any concern with the encountered interference, can only result in achieving the maximum throughout up to around 275 kbps. However, our projected algorithm has the capability of increasing the average throughput up

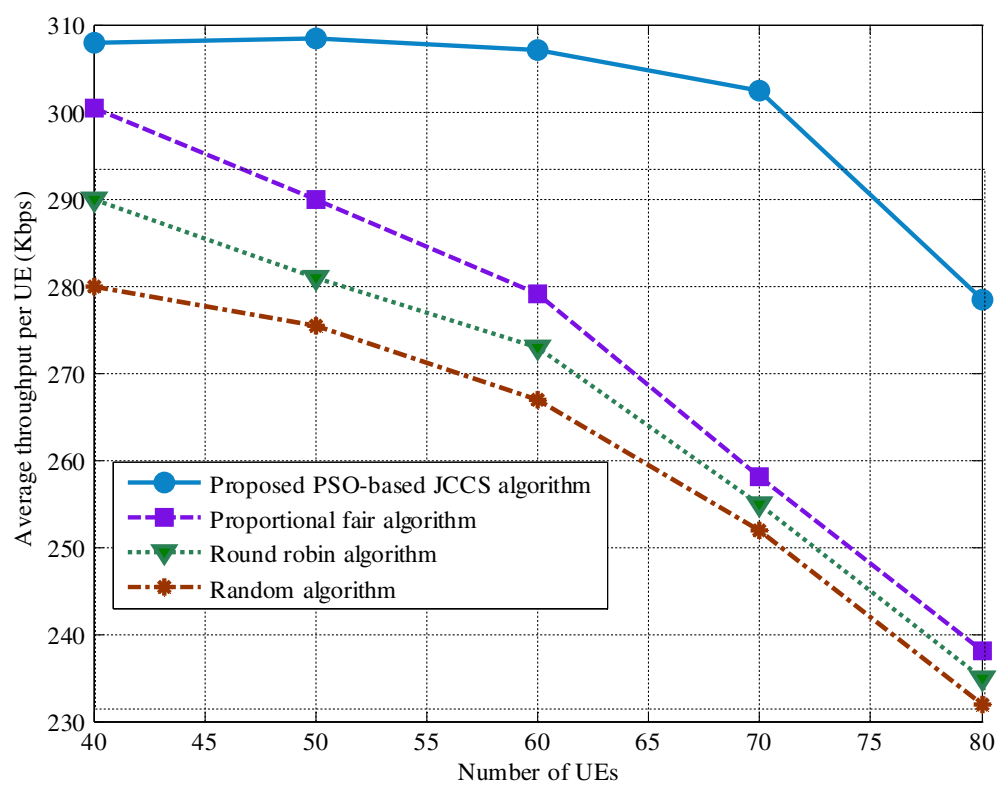

Figure 9 Throughput comparison. 


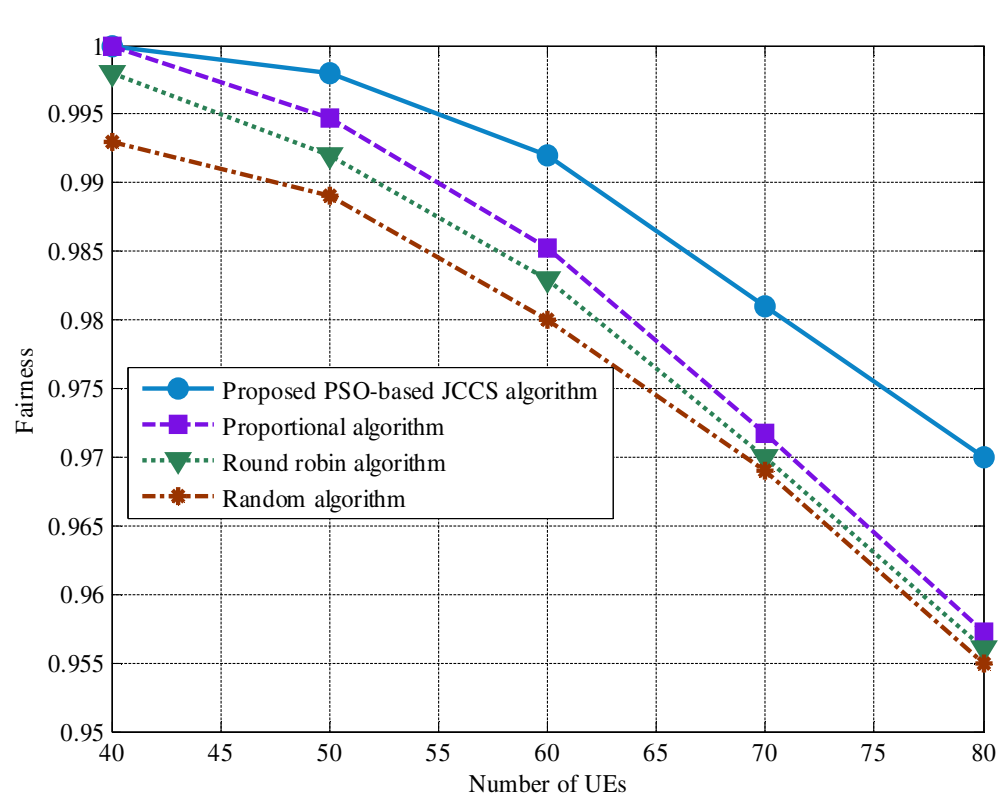

Figure 10 Fairness comparison.

to $350 \mathrm{kbps}$. Hence, it can be concluded that by employing PSO-based JCCS algorithm, the min-max throughput can significantly be enhanced.

\subsection{Comparison of PSO-based JCCS algorithm with traditional ones}

In this subsection of the analysis, we compared our proposed algorithm with the two well-known traditional CC selection and scheduling algorithms such as random, round robin, and proportional fair. The comparison is carried out in terms of throughput and fairness. Finally, the percentage gain of our proposed algorithm is also calculated in terms of throughput and fairness. For the sake of comparison, the best selected parameters as in section 6.2 are used for the proposed algorithms. The number of RBs and CCs, i.e., ( $M$ and $L$ ) are assumed to be fixed; however, the number of UEs $N$ are varied for getting a comparison.

\subsubsection{Throughput comparison}

Figure 9 illustrates the average throughput per UEs with respect to the number of UEs, $N$. Four curves are obtained by employing four selection/scheduling algorithms, i.e., proposed PSO-based JCCS algorithm, proportional fair, round robin, and random. It is shown that with the increase of a number of UEs per cell, throughput decreases, which is obvious because we have assumed a fixed number of RBs from the CC sets. However, by exploiting the PSObased JCCS algorithm, the throughput gap becomes wider as compared to the traditional methods by increasing the number of UEs. This is due to the fact that for fewer numbers of UEs, the ample availability of RBs results in a bit good average throughput by proportional fair, round robin, and random. On the other hand, the proportional fair performs better as compared to round robin for fewer numbers of UEs. This is in accordance to the fact that for users close to eNB, the proportional fair performs well as compared to round robin while its performance deteriorates for users at the cell edge. Thus, the performance gap between proportional fair and round robin reduces with the increase of UEs. However, for the proposed PSO-based JCCS, an algorithm performs well as compared to all the traditional algorithms. With the number of UE increases, the competition among the fixed number of RB increases and this results in the inferior performance of traditional algorithms compared with our proposed one. This is due to the fact that the PSO based-JCCS operates by considering the minimization of the inducted interferences. Moreover, there is about $20 \%$ improved per UE throughput after UE 70 with other traditional algorithms.

\subsubsection{Fairness comparison}

The comparison in terms of fairness of the proposed algorithm is illustrated in Figure 10. The parameter and fairness used to evaluate the performance of the algorithms is calculated by the fairness index (FI) formula in (15). The FI is a continuous value in the range between 0 and 1 . The larger index value means better system performance and vice versa.

$$
\mathrm{FI}=\frac{\left(\sum_{n=1}^{N} \gamma_{n}\right)^{2}}{N \times \sum_{n=1}^{N} \gamma_{n}^{2}}
$$




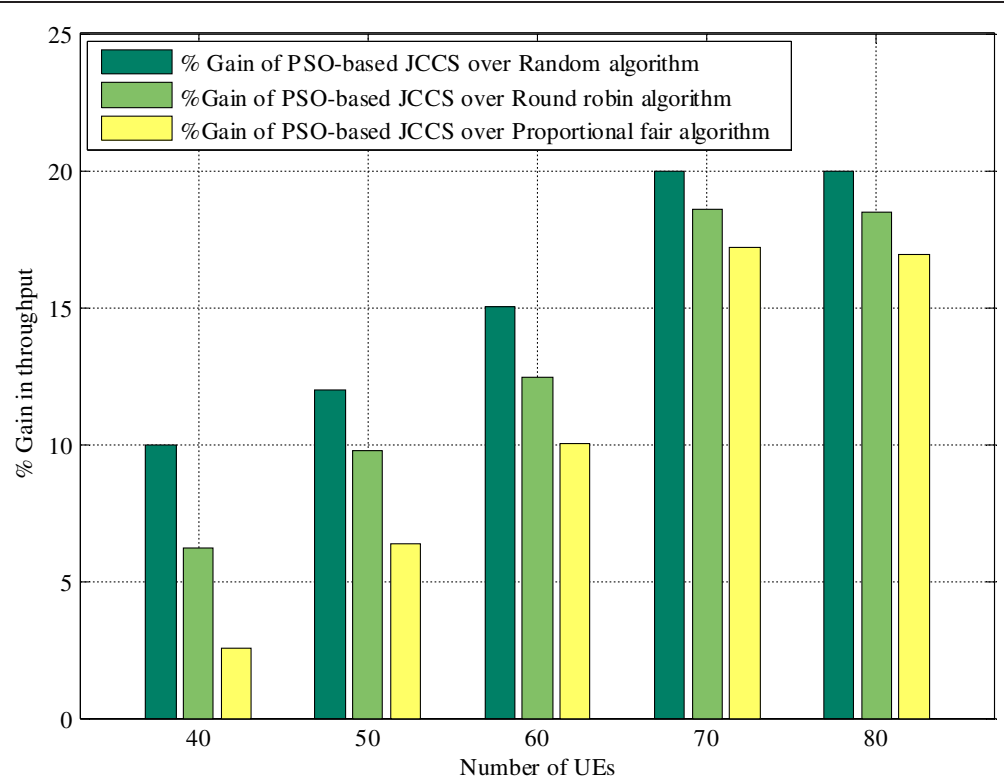

(a)

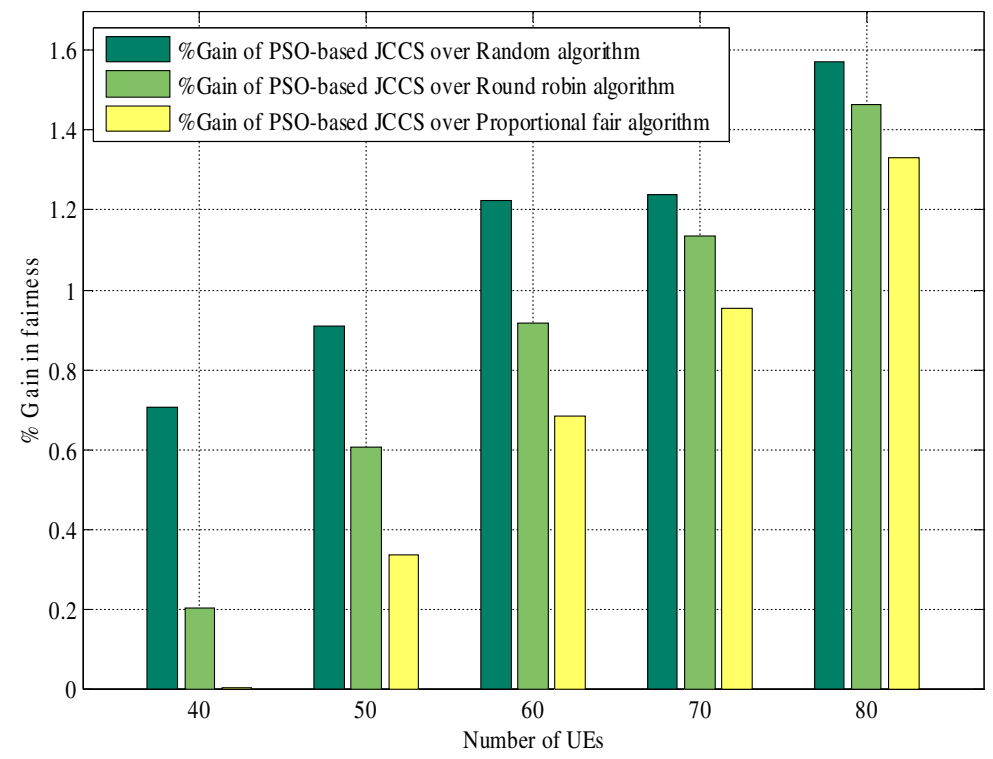

(b)

Figure 11 Percentage gain. (a) Throughput and (b) fairness.

where $N$ is the total UE to be serviced in the cell, and $\gamma_{n}$ is the average data rate of the $n$th UE. Figure 10 illustrates the comparison in terms of fairness with respect to the number of UEs of the CC selection and scheduling algorithms. As the fairness is dependent on per UE mean throughput, therefore, our proposed algorithm will definitely show better performance with respect to fairness also. It is also noted that with a fewer number of UEs, PSO-based JCCS algorithm shows same fairness as round robin and proportional fair because there is not much difference in the average throughput of the UEs; but as the number of UEs increases, the fairness curve gap becomes wider. This is due to the fact that PSO-based JCCS algorithm results in large per UE throughput because of optimum CC selection and RB assignments. Particularly, the performance of proportional fair is better than the other traditional methods for fewer numbers of UEs. However, this performance trend reduces with the increase of UEs. The reason being that the performance of proportional fair 
Table 2 System simulation parameters

\begin{tabular}{ll}
\hline Parameters & Setting (description) \\
\hline Site layout & Eight sites - three sectors per site - wrap around \\
Inter-site distance & $500 \mathrm{~m}$ \\
$\mathrm{TTI}$ & $1 \mathrm{~ms}$ \\
Path loss - LOS & $\mathrm{PL}=40 \log _{10}(d)+13.47-14 \log _{10}\left(h_{\mathrm{eNB}}\right)-14 \log _{10}\left(h_{\mathrm{UE}}\right)+14 \log _{10}\left(f_{C} / 5.0\right)$ \\
Path loss - NLOS & $\mathrm{PL}=\left(44.9-6.55 \log _{10}\left(h_{\mathrm{eNB}}\right)\right) \log _{10}(d)+31.46+5.83 \log _{10}\left(h_{\mathrm{eNB}}\right)+23 \log _{10}\left(f_{C} / 5.0\right)+40 \log _{10}(d)$ \\
Shadowing & Gaussian distribution with zero mean and $8 \mathrm{~dB}$ \\
Total eNB power & $46 \mathrm{dBm}$ \\
Antenna configuration & $1 \times 1$ \\
Thermal noise power & {$[3.5-4.5] \times 10^{-15} \mathrm{~W}$} \\
Traffic model & $\mathrm{Full}$ buffer \\
Component carriers & $4 \times 10 \mathrm{MHz}$ Contiguous @ $2 \mathrm{GHz}$ band. \\
Number of RBs & $50 \mathrm{RBs}$ per CC, $200 \mathrm{RBs}$ in total \\
Total UEs in system & $40-80$ \\
CQl frequency domain resolution & $1 \mathrm{CQI}$ per 3 RBs \\
\hline
\end{tabular}

algorithm is better than round robin for the UE close to the eNB while it become worst for UEs that are on the edge of eNB. The same argument holds while comparing fairness of random allocation and the proposed algorithms. For fewer UEs, the difference is more pronounced because of the large difference between the average data rate between the two.

\subsection{Percentage gain}

Figure 11 elaborates the percentage gain of the PSObased JCCS algorithm over random, round robin, and proportional fair algorithms with respect to UEs. For both the throughput and fairness cases, the percentage gain increased as a function of UEs because PSObased JCCS algorithm shows significant improvement by increasing UEs. More precisely, the percentage gain increases from $10 \%$ to $20 \%$ for the random algorithm, $6 \%$ to $17 \%$ for the round robin algorithm, and $2 \%$ to $16 \%$ for proportional fair algorithm with the increase of UEs from 40 to 80 as in Figure 11a. The increasing trend observed is due to the fact that the proposed PSO-based JCCS takes into account the interference while allocating the RBs from the $\mathrm{CC}$ set.

Similarly, the percentage gain in terms of fairness is plotted in Figure 11b. It can be illustrated that the percentage gain is not so significant for fewer number of

Table 3 Parameters for PSO-based JCCS

\begin{tabular}{lc}
\hline Parameters & Values \\
\hline Number of particles & $4,8,12,20$ \\
Acceleration coefficient $\left(c_{1}\right.$ and $\left.c_{2}\right)$ & 2 \\
Inertia weight $(W)$ & 0.6 \\
Number of iterations & 1,500 \\
{$\left[-V_{\min }, V_{\max }\right]$} & {$[-\mathrm{N}, \mathrm{N}]$} \\
\hline
\end{tabular}

UEs; however, percentage gain of the proposed PSO-based JCCS algorithm becomes significant as compared to traditional methods. This is the concern of iterative JCCS, which result in better RB assignments. The better $\mathrm{CC}$ selection and RB assignment in PSO-based JCCS election algorithm results in profound performance gap for increased UEs.

\section{Conclusions}

In this study, we investigated the self-organized PSO-based JCCS algorithm for inter-cell interference management in LTE-Advanced. Inter-cell interference management is the most critical task in LTE-Advanced that limits the performance of the networks. On the other hand, $\mathrm{CA}$ is exploited as a novel solution for meeting the increased throughput demands of UEs. Within the context of CA, CC selection and scheduling is the most critical task and has significant impact on the system performance. In order to alleviate these problems, a self-organized PSO-based JCCS is proposed for the downlink in LTE-Advanced. The general theme of the proposed algorithm is that it relies on the CQI values from the UEs on the direction of eNB, and based upon the values PSO-based JCCS, is carried out for distributing the resources with the concern of maximizing the throughput. An important point is that each eNB relies on the local received information without any involvement of centralized entity. In addition, the minimum throughput is also inculcated for alleviating the greediness behavior. As far as the reduction of information exchange is concerned, a single CQI value is used for representing the group of RBs from the CC set. Hence, it significantly reduces the information exchange. The comparison of the proposed PSO-based JCCS with traditional algorithms is carried out regarding average 
throughput and fairness. The results validate the superior performance of the proposed algorithm.

The benefit of the proposed PSO-based JCCS algorithms is that each eNB operate in a self-organizing manner and rule out the RBs allocation from the CC set with the concern of minimizing the impact of inter-cell interference. In addition, the proposed algorithm is also scalable in the sense that each eNB is individually responsible for the self-organizing scheduling task. However, the algorithm relies on the information in the form of CQI on the RBs for carrying out the scheduling task. This issue is addressed in this study by reporting on the bunch of RBs. The proposed metaheuristic algorithm takes some time to converge, and when the convergence is achieve, it gives optimum performance in terms of throughput and fairness. The investigation carried out in this study can be extended by considering the small cells.

\section{Competing interest}

The authors declare that they have no competing interests.

\section{Acknowledgements}

This research was supported by the MKE (The Ministry of Knowledge Economy), Korea, under the Convergence-ITRC (Convergence Information Technology Research Center) support program (NIPA-2014-H0401-14-1006) supervised by the NIPA (National IT Industry Promotion Agency) and the Basic Science Research Program through the National Research Foundation of Korea (NRF) funded by the Ministry of Education, Science and Technology (2012009449).

\section{Author details}

'Department of Information and Communication Engineering, Sejong University, Seoul 143-747, Republic of Korea. ${ }^{2}$ Department of Electronics and Electrical Engineering, University of Engineering and Technology Taxila, Taxila 47050, Pakistan.

Received: 30 April 2014 Accepted: 3 October 2014 Published: 20 October 2014

\section{References}

1. G Yuan, X Zhang, W Wang, Y Yang, Carrier aggregation for LTE-advanced mobile communication systems. IEEE Commun. Mag. 48(2), 88-93 (2010)

2. 3GPP RP-080137, Proposed SID on LTE-Advanced, NTT DoCoMo, etc., 3GPP TSG RAN meeting \#39, 2008

3. KI Pedersen, F Frederiksen, C Rosa, H Nguyen, LGU Garcia, Y Wang, Carrier aggregation for LTE-advanced: functionality and performance aspects. IEEE Commun. Mag. 49(6), 89-95 (2011)

4. M Iwamura, K Etemad, MH Fong, R Nory, R Love, Carrier aggregation framework in 3GPP LTE-advanced [WiMAX/LTE Update]. IEEE Commun. Mag. 48(8), 60-67 (2010)

5. H Hafez, YA Fahmy, MM Khairy, LTE and WiMAX: performance and complexity comparison for possible channel estimation techniques. Int. J. Commun. Syst. 26(6), 792-805 (2013)

6. 3rd Generation Partnership Project, 3GPP LTE-Advanced, http://www.3gpp. org/lte-advanced

7. Next Generation Mobile Networks Group (NGMN), http://www.ngmn.org

8. L Lei, K Zheng, Performance evaluation of carrier aggregation for elastic traffic in LTE-Advanced systems. IEICE Trans. Commun. E92.B(11), 3516-3519 (2009)

9. L Chen, WW Chen, X Zhang, DC Yang, Analysis and simulation for spectrum aggregation in LTE-Advanced system, in 2009 IEEE Vehicular Technology Conference (VTC Fall) (IEEE, Alaska, 2009), pp. 1-6

10. L Zhang, YY Wang, L Huang, HL Wang, WB Wang, QoS performance analysis on carrier aggregation based LTE-A systems, in IET International Communication Conference on Wireless Mobile and Computing (CCWMC) (IET, Shanghai, 2009), pp. 253-256
11. YY Wang, KI Pedersen, TB Sorensen, PE Mogensen, Carrier load balancing and packet scheduling for multi-carrier systems. IEEE Trans. Wireless. Commun. 9(5), 1780-1789 (2010)

12. YY Wang, KI Pedersen, PE Mogensen, TB Sorensen, Resource allocation considerations for multi-carrier LTE-Advanced systems operating in backward compatible mode, in 2009 IEEE $20^{\text {th }}$ International Symposium on Personal, Indoor and Mobile Radio Communications (IEEE PIMRC) (IEEE, Tokyo, 2009), pp. 370-374

13. LGU Garcia, KI Pedersen, PE Mogensen, Autonomous component carrier selection: interference management in local area environments for LTE-advanced. IEEE Commun. Mag. 47(9), 110-116 (2009)

14. H Wang, C Rosa, K Pedersen, Performance analysis of downlink inter-band carrier aggregation in LTE-Advanced, in 2011 IEEE Vehicular Technology Conference (VTC Fall) (IEEE, San Francisco, 2011), pp. 1-5

15. H Tian, S Gao, J Zhu, L Chen, Improved component carrier selection method for non-continuous carrier aggregation in LTE-Advanced System, in 2011 IEEE Vehicular Technology Conference (IEEE VTC Fall) (IEEE, San Francisco, 2011), pp. 1-5

16. LGU Garcia, IZ Kovacs, KI Pedersen, GWO Costa, PE Mogensen, Autonomous Component Carrier Selection for 4G Femtocells - A Fresh Look at an Old Problem. IEEE J. Sel. Areas. Commun. 30(3), 525-537 (2012)

17. A Vavooulas, N Vaipoulos, DA Varoutas, A Chipouras, G Stefanou, Performance improvement of fixed wireless access networks by conjunction of dual polarization and time domain radio resource allocation technique. Int. J. Commun. System. 24(4), 483-491 (2011)

18. L Bai, C Chen, B Wu, J He, Adaptive resource allocation for multicast orthogonal frequency division multiple access systems with guaranteed BER and rate. Int. J. Commun. System. 26(7), 912-926 (2013)

19. C Chen, F Baccelli, Self-optimization in mobile cellular networks: power control and user association, in 2010 IEEE International Conference on Communications (IEEE ICC) (IEEE, Cape Town, 2010), pp. 1-6

20. M Aydin, R Kwan, J Wu, Multiuser scheduling on the LTE downlink with meta-heuristic approaches. Phys. Commun. 9, 257-265 (2013)

21. A Shahid, S Aslam, KG Lee, A decentralized heuristic approach towards resource allocation in femtocells networks. Entropy 15(7), 2524-2547 (2013)

22. A Shahid, S Aslam, HS Kim, KG Lee, A docitive Q-learning approach towards joint resource allocation and power control in self-organized femtocells networks. Trans. Emerg. Telecommunication Technol. (2014). doi:10.1002/ett.2800

23. H Nguyen, IZ Kovacs, Downlink Radio Resource Management for LTE-Advanced System with Combined MU-MIMO and Carrier Aggregation Features, in 2012 IEEE Vehicular Technology Conference (IEEE VTC Spring) (IEEE, Yokohama, 2012), pp. 1-5

24. E Dahlman, S Parkvall, J Skold, P Beming, $3 \mathrm{~g}$ HSPA and LTE for Mobile Broadband (Academic Press, New York, 2007)

25. Evolved universal terrestrial radio access E-UTRA, Physical channels and modulation, release 8. Technical Report 3G TS36.211, 3rd Generation Partnership Project (3GPP, New Orleans, 2007)

26. J Kennedy, RC Eberhart, Particle swarm optimization, in IEEE International Conference on Neural Networks (Perth, 1995), pp. 39-43

27. H Izakian, A Abraham, V Snášel, Metaheuristic Based Scheduling Meta-Tasks in Distributed Heterogeneous Computing Systems. Sensors 9(7), 5339-5350 (2009)

28. F Solis, R Wets, Minimization by Random Search Techniques. Math. Oper. Res. 6, 19-30 (1981)

29. P Kyösti, J Meinila, L Hentila, X Zhao, T Jamsa, C Schneider, M Narandzic, M Milojevic, A Hong, J Ylitalo, V-M Holappa, M Alatossava, R Bultitude, Y de Jong, T Rautiainen, IST-4-027756 WINNER II D1. 1.2 V1. 1 WINNER II Channe Models. Inf. Soc. Technol. 11, (2007). Forschungsbericht

30. Y Shi, RC Eberhart, Parameter selection in particle swarm optimization, in 7th Annual Conference on Evolution Programming VII (Springer, San Deigo, 1998), pp. 591-601

doi:10.1186/1687-1499-2014-171

Cite this article as: Shahid et al:: A self-organized metaheuristic approach towards inter-cell interference management for LTE-Advanced. EURASIP Journal on Wireless Communications and Networking 2014 2014:171. 